\title{
Analysis of the interaction and proliferative activity of adenocarcinoma, peripheral blood mononuclear and mesenchymal stromal cells after co-cultivation in vitro
}

\author{
Kristina V. Kitaeva ${ }^{1}$, Tikhon S. Prudnikov ${ }^{1}$, Marina O. Gomzikova ${ }^{1}$, Sevindzh K. Kletukhina ${ }^{1}$, Victoria \\ James $^{2}$, Albert A. Rizvanov ${ }^{1}$, Valeriya V. Solovyeva ${ }^{1}$ \\ ${ }^{1}$ Institute of Fundamental Medicine and Biology, Kazan Federal University, Kazan, Russia \\ ${ }^{2}$ School of Veterinary Medicine and Science, University of Nottingham, Nottingham, United Kingdom
}

\begin{abstract}
The tumor microenvironment is a heterogeneous population of cells actively involved in the process of growth and development of a tumor. Research has demonstrated the interactions between the different populations of cells are critical for the formation of the tumour micro-environment and if recapitulated experimentally can be used to produce more effective models for preclinical screening of anticancer drugs. In this study, we demonstrate co-culturing HeLa adenocarcinoma cells, peripheral blood mononuclear cells and mesenchymal stromal cells, results in changes in the proliferative activity of the peripheral blood mononuclear cells and mesenchymal stromal cell populations. This data supports the further development of in vitro co-culture systems utilizing these cell types for pre-clinical screening of anticancer drugs.
\end{abstract}

Keywords: co-culture, intercellular interaction, adenocarcinoma, mononuclear blood cell, mesenchymal stromal cell, test system, drug screening

\section{Introduction}

The microenvironment plays a key role in tumor progression, metastasis and the formation of therapeutic resistance [1]. The tumor stroma consists of a mixed-cellular component that includes immune cells, endothelial cells, tumor-associated fibroblasts and myofibroblasts, adipocytes and mesenchymal stromal cells (MSCs), as well as an extracellular matrix that provides structural support for the cell component in the extracellular space and organs [2]. The recruitment of MSCs into the tumor stroma is generally as a result of inflammatory factors produced within the tumor microenvironment [3]. After recruitment to the tumor environment, MSCs can act as precursors of cancer-associated fibroblasts (CAFs), which contribute to the progression of tumors due to the secretions of a number of growth factors, chemokines, interleukins, and matrix metalloproteinases [4]. The migratory response of MSC's to soluble factors such as MIP- $1 \delta$ and MIP- $3 \alpha$, is evidence of this ability to respond to tumor secreted factors [5]. Using bioluminescent imaging, it has been shown that MSCs after administration to mice within a xenograft model of human glioma, localize to areas of tumor formation and are not detected in healthy areas of the brain [6,7]. At the same time, fibroblasts did not show similar specificity of the ability to migrate, which indicates the selective migration of MSCs to the area of tumor formation [6].

MSCs are involved in the normal tissue regeneration processes, migrating to sites of damage in response to inflammatory mediators. This same process occurs within tumors that express a similar repertoire of factors as the inflamed tissue [1]. Stromal cells attracted by tumor secrete growth factors of various types, such as hepatocyte growth factor (HGF), fibroblast growth factor (FGF), vascular endothelial growth factor (VEGF), transforming growth factor $\beta$ (TGF- $\beta$ ), epidermal growth factor (EGF) [8] and chemokines, including CXCL12, not only promote the growth and survival of tumor cells, but also act as a chemoattractant for other cells to migrate into the microenvironment of the tumor. In addition, MSCs actively remodel the extracellular matrix, contribute to neoangiogenesis and the formation of resistance to chemotherapeutic drugs [3]. In general it is assumed the changes induced by MSCs collectively result in a pro-tumor growth environment, as illustrated by the incorporation of MSCs as part of both 3D in vitro and in vivo models of human glioblastoma, resulting in an increase in both the proliferative and invasive potential of tumor cells [9]. However, MSCs have been reported to inhibit tumor growth, by attracting cells of the inflammatory response, negatively affecting angiogenesis and the Wnt and AKT signaling pathways, inducing cell cycle arrest and apoptosis [10,11]. The mechanisms underpinning these differential effects on the tumor microenvironment are yet to be fully understood, but may depend on the activation status of these cells and other elements of the stromal microenvironment of the tumor. For example, MSCs isolated from adipose tissue of mole rats (Blind Mole Rat Spalax) showed antitumor activity and reduced migration activity to tumor sites, in contrast to MSCs isolated from adipose tissue of ordinary rats [12]. Despite the multitude of tumor-supporting properties, the ability of MSCs to migrate and reside within the tumor environment makes this cell type a promising vector for the delivery of therapeutic gene products, chemotherapeutic drugs, and immunomodulating agents [1,13].

In addition to changes caused by MSCs, the tumor microenvironment can also directly and indirectly disrupt the normal function of infiltrating immune cells [14]. Tumor cells and tumor-associated stromal cells express inhibitors of the immune response control points that suppress the function of T-cells, through PD-1 and its ligand (PD-L1), and inhibitors of CTLA-4, which contributes to the avoidance of immune surveillance in both solid and hematological tumors [15]. In addition to direct immunosuppression using regulatory ligands, tumor cells also coopt microorganism that produce high levels of lactate or kinurenin, which directly inhibit T-cells [16]. In addition, 
some populations of immune cells, in particular, T-regulatory cells, are able to exert an immunosuppressive effect on T-effector cells [17].

The tumor-recruited macrophages of the M2-phenotype form a significant contribution to the development of the tumor and suppression of the antitumor immune response. It is known that a decrease in an excessive immune response by M2-macrophages promotes more active tumor growth and its escape from immune surveillance. M2macrophages respond to cytokines IL-4 and IL-13, which leads to activation of the transcription factor STAT6 and activation of the signal anti-inflammatory genes arginase (Arg1) and resistin-like molecule $\alpha$ [18]. These regulatory pathways contribute to the recruitment of immunoregulatory macrophages, the release of immunoregulatory cytokines, the induction of angiogenesis and the remodeling of basement membranes [19].

Studies of the tumor microenvironment in recent years have shown the need to develop new test systems that can take into account the factors of the microenvironment and the heterogeneity of the natural tumor [20,21]. However, the creation of a model that is able to take into account all aspects of the microenvironment is not currently possible. Nevertheless, the strategy of using several basic elements involved in the tumor formation processes can significantly improve the prognostic value of such test-systems compared to conventional single cell tumor models [22]. Thus, to reproduce the processes occurring in the stromal microenvironment of a tumor, the development of in vitro test systems containing several cellular components of the tumor microenvironment is required.

The study reports the beneficial interactions of co-culturing adenocarcinoma cells with peripheral blood mononuclear cells and mesenchymal stromal bone marrow cells in vitro. This data highlights the value of including these and similar cell types into complex pre-clinical models for cancer drug testing.

\section{Materials and methods}

\section{Isolation and cultivation of cells}

Bone marrow samples were provided by the Republican Clinical Hospital for research purposes in accordance with ethical standards and current legislation (the protocol was approved by the Committee on Biomedical Ethics of Kazan Federal University (No. 3, 03/23/2017)). Written informed consent was obtained from donors. MSCs from human bone marrow were isolated in a ficoll density gradient $\left(1.077 \mathrm{~g} / \mathrm{cm}^{3}\right.$, PanEco, Russia), followed by adhesion of cells to the surface of culture plastic. Human adenocarcinoma cells (HeLa) were obtained from the American Type Culture Collection (ATCC number: CCL2, Manassas, VA, USA). Mononuclear cells were isolated from peripheral blood (PBMCs) of a healthy donor in a ficoll density gradient $\left(1.077 \mathrm{~g} / \mathrm{cm}^{3}, \mathrm{PanEco}\right.$, Russia) as previously described [23,24]. Written informed consent was obtained from donors. MSCs, HeLa and PBMCs were cultured in DMEM/F12 (PanEco, Russia) supplemented with 10\% FBS (Invitrogen, USA), 2 mM Lglutamine (PanEco, Russia), penicillin (100 U/ml) and streptomycin $(100 \mu \mathrm{g} / \mathrm{ml})$ (Biolot, Russia). Cells were maintained at $37{ }^{\circ} \mathrm{C}$ in a humid atmosphere with a content of $5 \% \mathrm{CO}_{2}$. Sub-confluent cells (approximately $80 \%$ confluent) were passaged using $0.25 \%$ trypsin-EDTA solution (Invitrogen, USA).

\section{Immunophenotyping of peripheral blood mononuclear cells (PBMC)}

PBMC immunophenotyping was performed using the following CD3 antibodies (Cat. No. 300405, BioLegend, USA), CD4 (Cat. No. 317409, BioLegend, USA), CD8 (Cat. No. MA5-16988, Thermo Fisher Scientific, USA), CD14 (Cat. No. 301813, BioLegend, USA), CD34 (Cat. No. 345802, BD Biosciences, USA), CD45 (Cat. No. 304009, BioLegend, USA). The cell population was analyzed using FACS Aria III (BD Biosciences, USA) and BD FACSDiva ${ }^{\mathrm{TM}}$ software version 7.0.

\section{Immunophenotyping of mesenchymal stem cells (MSC) isolated from bone marrow}

MSC immunophenotyping was performed using the following antibodies to CD29 (Cat. No. 87106, SONY, USA), CD44 (Cat. No. 51-9007656, BD Biosciences, USA), CD73 (Cat. No. 51-9007649, BD Biosciences, USA), CD90 (Cat. No. 51-9007657, BD Biosciences, USA), CD105 (Cat. No. 323218, BioLegend, USA), CD166 (Cat. No. 10846, SONY, USA), a cocktail of conjugated PE antibodies hMSC Negative Cocktail potential contaminants from the Human MSC Analysis Kit (Cat. No. 562245, BD Biosciences, USA) was used as a negative control. The staining results were analyzed using FACS Aria III (BD Biosciences, USA) and BD FACSDiva ${ }^{\mathrm{TM}}$ software version 7.0.

\section{Fluorescent staining of cells}

Fluorescent staining of MSCs, HeLa and PBMCs was performed in a serum-free medium using the Vybrant Cell Labeling Solutions kit (Thermo Fisher Scientific, USA) [25] using the dyes DiD (red spectrum), DiI (yellow spectrum) and $\mathrm{DiO}$ (green spectrum), respectively, according to the method recommended by the manufacturer. A qualitative assessment of staining was performed on an AxyObserver.Z1 fluorescence microscope (Carl Zeiss, Germany) and using the software AxyoVision Rel. 4.8.

Co-cultivation of cells

For co-culture, fluorescently stained MSCs, HeLa and PBMCs were mixed in a 1:1:1 ratio in a 12-well plate $\left(6 \times 10^{4}\right.$ cells/well). MSCs, HeLa and PBMCs were co-cultured in DMEM/F12 (PanEco, Russia) supplemented with 10\% FBS (Invitrogen, USA), 2 mM L-glutamine (PanEco, Russia), penicillin (100 U/ml) and streptomycin 
$(100 \mu \mathrm{g} / \mathrm{ml})$ (Biolot, Russia). Cells were maintained at $37{ }^{\circ} \mathrm{C}$ in a humid atmosphere with a content of $5 \% \mathrm{CO}_{2}$. The self-organization of co-culture cells was analyzed using a fluorescence microscopy on an AxyObserver.Z1 microscope (Carl Zeiss, Germany) and using the software AxyoVision Rel. 4.8.

\section{FACS analysis}

After 72 hours of co-culture, the cells were trypsinized and washed using Dulbecco's phosphate-buffered saline solution (PanEco, Russia). The cells were then sorted according to the fluorescence spectrum and size on a FACS Aria III flow cytometer (BD Biosciences, USA) using BD FACSDiva ${ }^{\mathrm{TM}}$ software version 7.0.

\section{Analysis of cell proliferation activity}

After separation, individual cell populations of MSCs, HeLa and PBMCs were sown in a 96-well plate and cultured for 24 hours in DMEM/F12 (PanEco, Russia) supplemented with 10\% FBS (Invitrogen, USA), 2 mM Lglutamine (PanEco, Russia), penicillin $(100 \mathrm{U} / \mathrm{ml})$ and streptomycin $(100 \mu \mathrm{g} / \mathrm{ml})$ (PanEco, Russia) at $37^{\circ} \mathrm{C}$ in a humid atmosphere with a content of $5 \% \mathrm{CO}_{2}$. CellTiter $96^{\circledR}$ AQueous Non-Radioactive Cell Proliferation Assay (Promega, USA) was used to determine cell proliferation according to the method recommended by the manufacturer.

\section{Statistical analysis}

The analysis of the obtained data was carried out using the GraphPad Prism 7 software (GraphPad Software) using the Shapiro-Wilk criterion and the Student's t-test.

\section{Results and discussion}

Mononuclear cells were isolated from the peripheral blood of a healthy donor. Immunophenotypic analysis of human PBMCs showed the presence of a large population (82.6\%) of cells carrying a CD45 leukocyte marker on their surface (Figure 1B), among which $81.8 \%$ were T-lymphocytes (CD3 +, CD45 +) (Figure 1C), of which33.4\% were T-cytotoxic lymphocytes (CD3+, CD8+, CD45+) and 44.1\% T-helper cells (CD3+, CD4+, CD45+) (Figure 1D), together with $0.9 \%$ hematopoietic progenitor cells (CD34+ and CD45+) (Figure 1E) and 2.2\% CD14+ monocytes (Figure 1F), consistent with published data [26]. It is known that T-cell populations are actively involved in the development of a cellular immune response to cancer, as shown in the study of the microenvironment of colorectal cancer, melanoma and breast cancer, in which the high content of infiltrate activated CD8+/CD4+ T cells in the early stages is a positive prognostic sign for patients [27-30]. In addition, cells carrying a CD14 marker on their surface can differentiate into macrophages with M1 and M2 phenotypes; involved in both the development and suppression of the immune response.
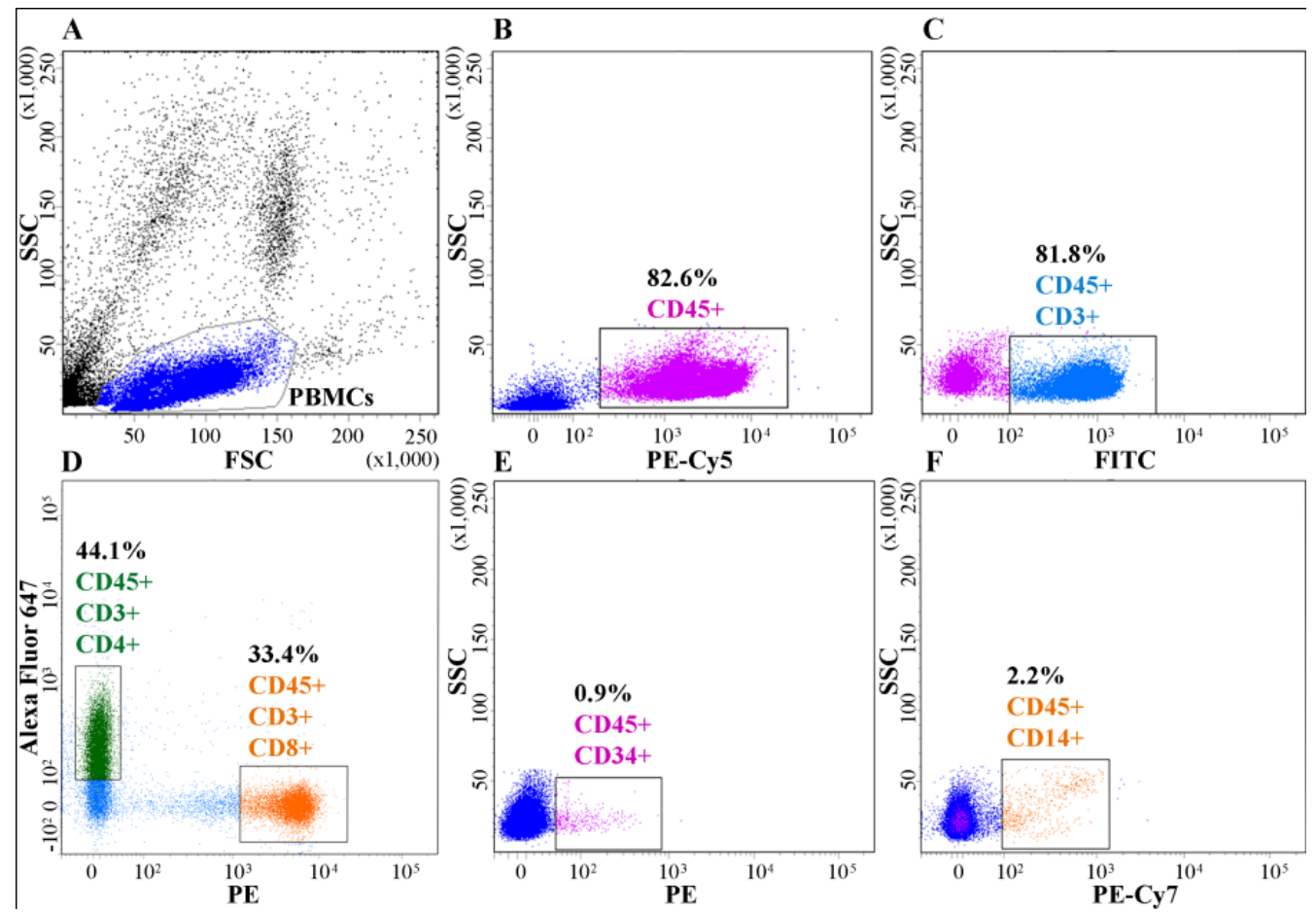

Figure 1. Immunocytometric characterisation of surface antigens (CD-markers) on isolated PBMCs. (A) The total PBMC population. (B-F) Population of cells carrying the leukocyte marker (B) CD45 (82.6\%). (C) CD3+/CD45+ T-lymphocytes (81.8\%). (D) CD3+/CD4+/CD45+ T-helper (44.1\%), CD3+/CD8+/CD45+ Tcytotoxic lymphocytes (33.4\%). (E) CD34+/CD45+ hematopoietic progenitor cells (0.9\%). (F) CD14+/CD45+ monocytes $(2.2 \%)$. 
The MSCs isolated from bone marrow had a mesenchymal stromal cell phenotype, expressing surface markers CD90, CD166, CD29, CD73, CD44, CD105, lacking surface markers characteristic of hematopoietic cells (CD11b, CD19, CD34, CD45, HLA-DR) (Figure 2).
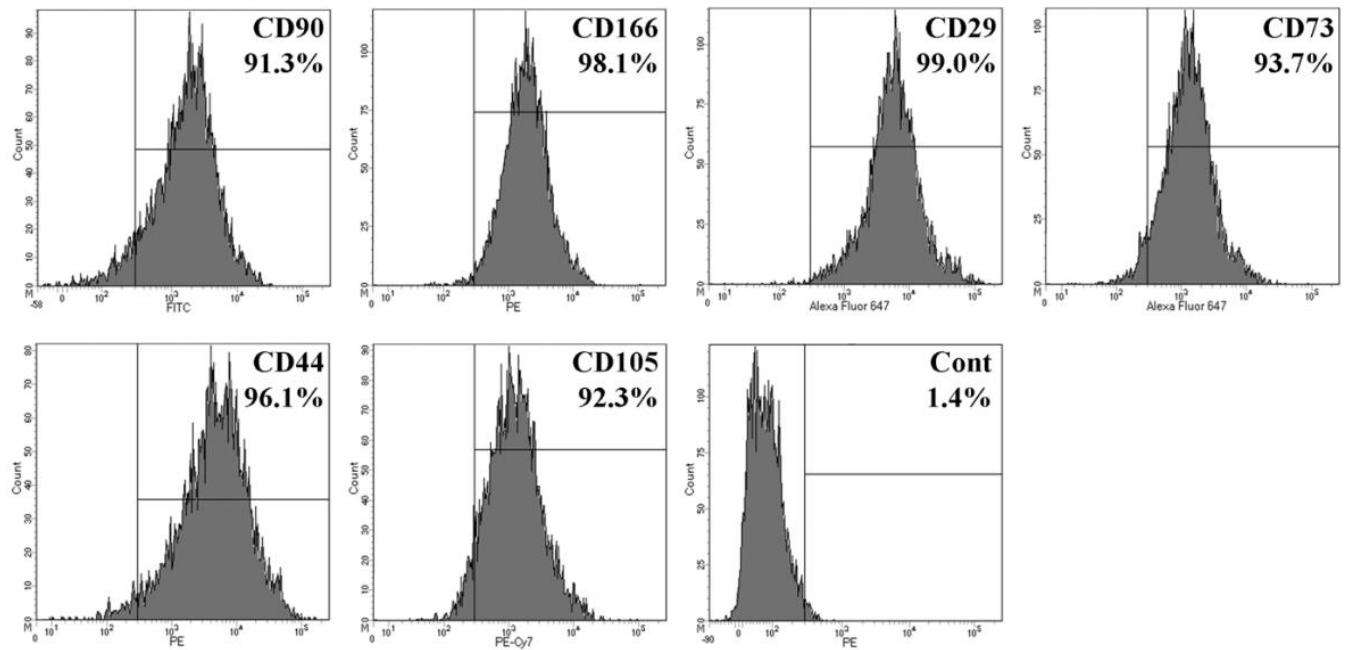

Figure 2. Immunocytometric characterization of CD surface antigens CD90, CD166, CD29, CD73, CD44, CD105. Cont - negative marker cocktail CD11b, CD19, CD34, CD45 and HLA-DR.

To analyze the behavior of adenocarcinoma cells, PBMCs and bone marrow MSCs were co-cultures with HeLa adenocarcinoma cells. Vital fluorescent staining of cells was carried out and tracing to determine the selforganization of individual cell populations in the tumor microenvironment model was conducted. The approach is based on the previously developed technology for creating pseudo-two-dimensional models of the interaction of tumor and stem cells using various extracellular matrices [25]. The cells were subsequently separated by cell-type by FACS.

The co-culture of MSCs, HeLa adenocarcinoma cells and PBMCs, revealed a high-degree of interaction between the cells types. Figure 3D, demonstrates cells with mixed fluorescence spectra (indicated by arrows), this potentially demonstrates an active exchange of membrane components, or the fusion and formation of hybrid cells. Similar phenomena are described in the literature [31,32]. The potential phenomena of tumour-stroma cell fusions and uptake of tumor cell fragments by immune cells and vice-versa, would contribute significantly to the heterogeneity of cells within the tumor and environment as we all potentially contributing to suppression of the immune response and the formation of resistance to chemotherapeutic drugs [33,34].
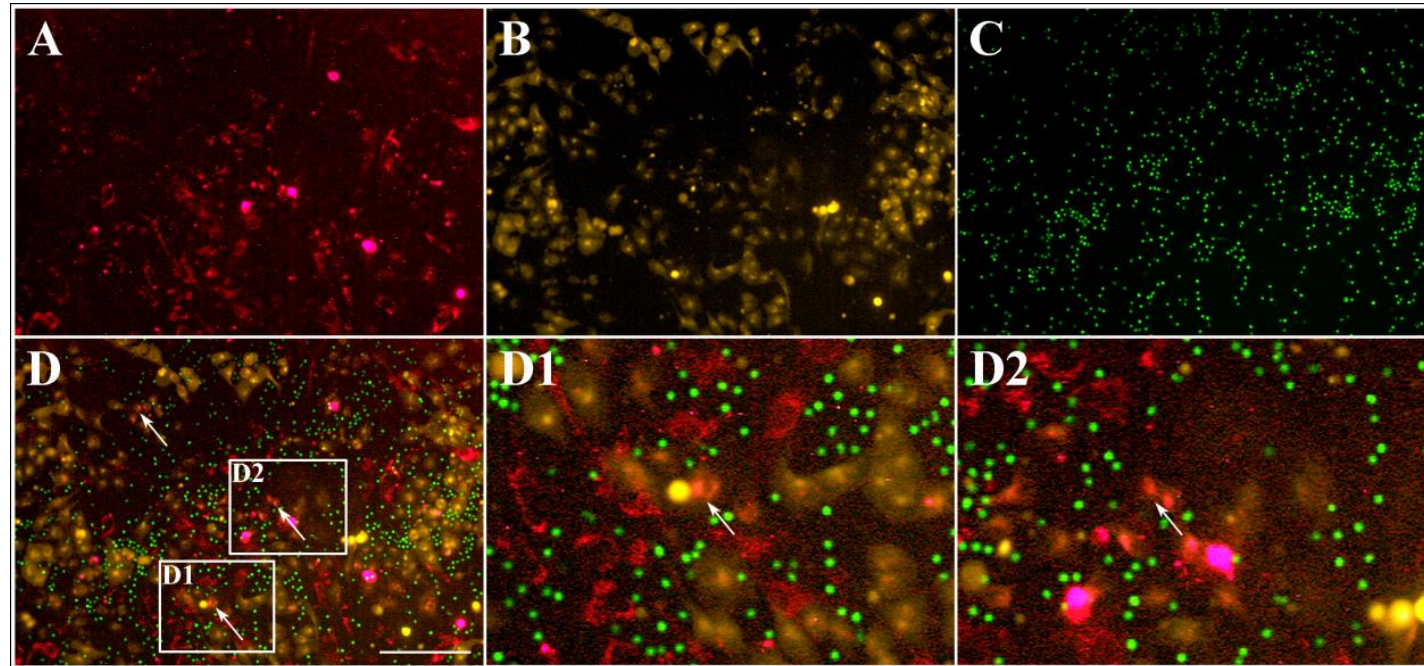

Figure 3. Analysis of self-organization of MSCs, Hela and PBMCs after 72 hours of co-culture. Arrows indicate fused cells. A - Alexa Fluor 647 (MSCs), B - PE (HeLa), C - FITC (PBMCs), D, D1, D2 - merge of Alexa Fluor 647, PE and FITC spectra. Scale: 200 microns.

After 72 hours of co-cultivation, cytofluorimetric analysis showed a high degree of cell interaction through the exchange of membrane components, which was expressed as the mixing of fluorescence spectra. The highest percentage of mixed fluorescent spectra were observed between MSCs and PMBCs (Alexa Fluor 647 and FITC) $47.9 \%$ (Figure 4B), a smaller percentage of exchanged membrane components was observed between MSCs and 
HeLa (Alexa Fluor 647 and PE) (Figure 4A) - 18.5\%, and between HeLa and PBMCs (PE and FITC) (Figure 4C) $5.7 \%$, while the percentage of PBMCs that did not interact with both MSCs and HeLa cells accounted for about $5.6 \%$ (Figure 4C). To note, a relatively high percentage of cells lost the fluorescent labels during the co-cultivation process - overall decrease by $24.1 \%$ (HeLa and MSCs), 69.9\% (PBMCs and MSCs) and 18.8\% (HeLa and PBMCs) in total number of fluorescent cells in both cell populations in co-culture (Figure 4A, B, C). The active interaction of cells with each other and the exchange of membrane components can be explained by extracellular vesicle transport and direct cell-to-cell contacts. A recent study was showed the transfer of vesicle material from prostate cancer cells to bone marrow derived stromal cells [35]. It is known that extracellular vesicles transfer components such as proteins, nucleic acids (DNA, RNA), non-coding RNA, as well as proteins [36]. Due to this content, the phenomenon of extracellular communication between cells of the tumor microenvironment contributes to the development of resistance to chemo-and radiotherapy as well as contributing to tumor progression [37]. However, in addition to negative effects, promising options for the use of microvesicles for therapeutic purposes have been shown in a number of publications [38]. In addition, it has been shown in the literature that when co-cultivated, it is possible to transfer functional mitochondria from mesenchymal stromal cells to tumor cells. This process led to increased oxidative phosphorylation in tumor cells and subsequent increases in their proliferative and invasive ability [39].
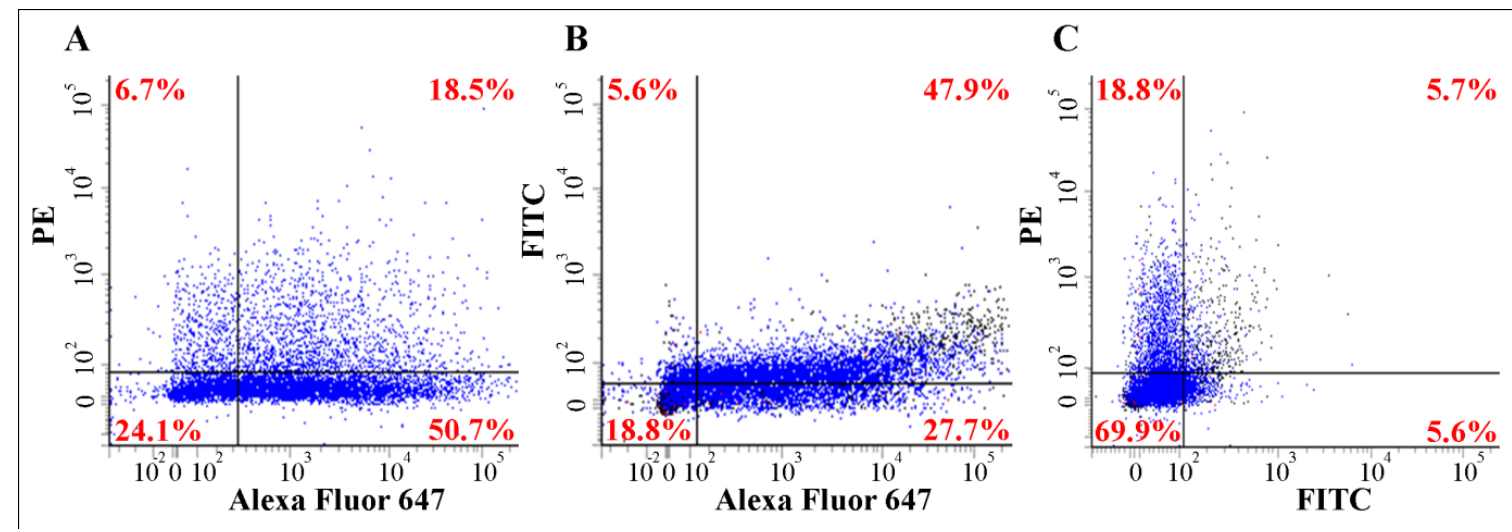

Figure 4. Cytofluorimetric analysis of individual cell populations of HeLa (PE), MSCs (Alexa Fluor 647) and PBMCs (FITC) after 72 hours co-culture. Analysis of cell populations from the fluorescence spectra of (A) PE and Alexa Fluor 647 (HeLa and MSCs); (B) FITC and Alexa Fluor 647 (PBMCs and MSCs); (C) PE and FITC (HeLa and PBMCs).

To analyze the effect of co-culture on the proliferation of the individual cell populations, following 72 hours co-culture cells were sorted based on their fluorescence spectra and the proliferation of each sorted cell population determined. The effect of co-culture on the proliferation of MSCs was significantly increased (more than 2 -fold, $\mathrm{p}<0.0001)$. This result can be explained by the response of MSCs to inflammatory cytokines secreted by coculture cells, which leads to the induction of their proliferation [3].

At the same time, a noticeable decrease in the proliferation of PBMCs was shown, namely by $62 \%$ (p < 0.0001) (Figure 5). The results obtained are consistent with experimental data from the literature, which showed that the tumor microenvironment, which is rich in inflammatory cytokines, suppresses antitumor immune functions, preventing the recruitment, survival and functioning of immune effector cells [40,41]. In addition, early research showed MSCs exert an immunosuppressive effect both by paracrine regulation and by direct cellular interactions [42]. It has been shown that MSCs have a suppressive effect on populations of CD3+, CD4+ and CD8+ subpopulations of T-lymphocytes [43].

A slight decrease (by 10\%, p < 0.05) of the proliferation of HeLa cells after co-culture with MSCs and PBMCs (Figure 5) is also shown. It is possible that MSCs and PBMCs under these culture conditions had a slight inhibitory effect on the growth of HeLa adenocarcinoma cells, which can be explained by the high density of the cell monolayer and the presence in the culture medium of a large number of different metabolites. In addition, it is known that MSCs can have antitumor effects under certain conditions and depending on the source of origin [12]. Further research is needed to fully understand the mechanisms that lead to the inhibition of the growth of HeLa adenocarcinoma cells in co-culture with MSCs and PBMCs. 

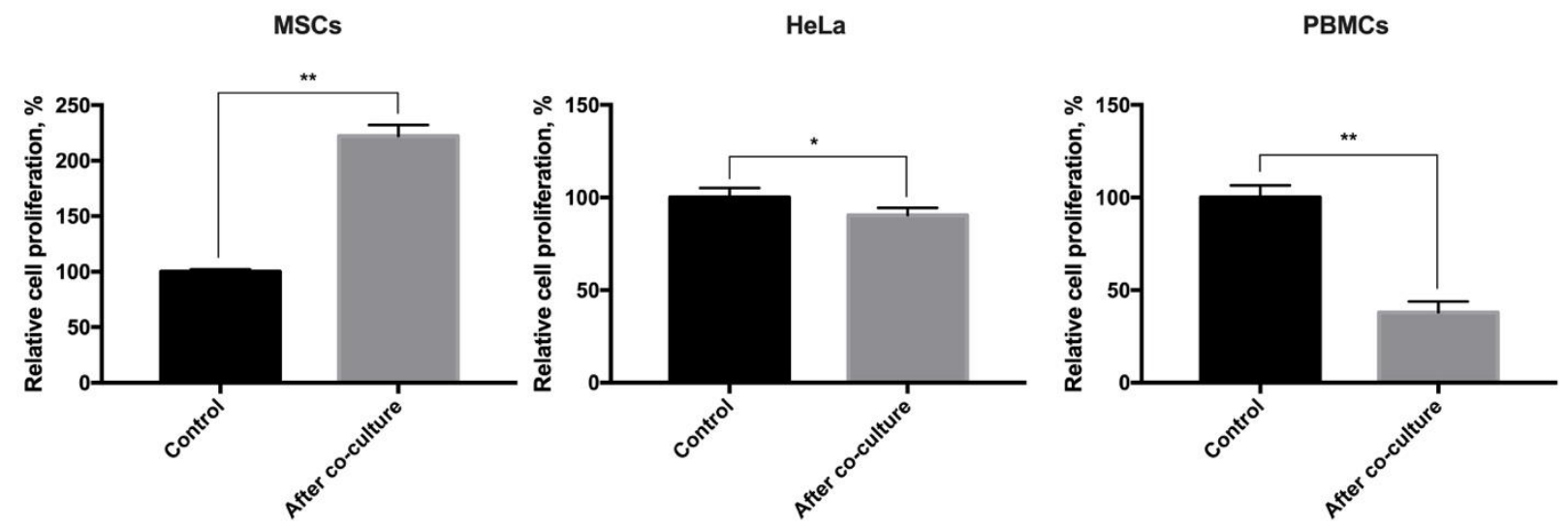

Figure 5. Analysis of changes in proliferation of the individual cell populations following culture as individual cell types (control) or after 3D-co-culture (HeLa, MSCs and PBMCs).

$$
*-\mathrm{p}<0.05, * *-\mathrm{p}<0.0001 \text {. }
$$

\section{Conclusions}

The results of the study showed that HeLa adenocarcinoma cells, PBMCs and bone marrow derived MSCs in co-culture actively interact with each other not only by paracrine regulation, but also potentially by direct cell-tocell contact, extra-cellular vesicular transport and fusion into hybrid cells. The co-culture of the three cell types together, resulted in modulation of the viability of MSCs and PBMCs, likely due to the intercellular interactions and exposure to various biologically active molecules and metabolites secreted within the co-culture environment. A possible antitumor effect of MSCs and PBMCs on adenocarcinoma cells is also described. However, it is currently unclear if this is due to the origin of the cell types within the co-culture e.g. MSCs derived from the bone marrow or the physical parameters of the culture system e.g. the high-density of cells. Therefore, further studies are needed to confirm the antitumor effects described. Together the data generated supports the active interaction of adenocarcinoma cells, MSCs and PBMCs when co-cultured, confirming the use of multi-cell type co-cultures is not only a relevant approach, but also essential for the development of potential in vitro preclinical screening systems for antitumor drugs.

\section{Acknowledgments}

This study was supported by the Russian Science Foundation grant 18-74-10044. The work is performed according to the Russian Government Program of Competitive Growth of Kazan Federal University and subsidy allocated to Kazan Federal University for the state assignment in the sphere of scientific activities.

\section{References}

1. Chulpanova DS, Kitaeva KV, Tazetdinova LG, James V, Rizvanov AA, Solovyeva VV (2018) Application of Mesenchymal Stem Cells for Therapeutic Agent Delivery in Anti-Tumor Treatment. Front Pharmacol 9. doi:10.3389/fphar.2018.00259

2. Valkenburg KC, de Groot AE, Pienta KJ (2018) Targeting the tumour stroma to improve cancer therapy. Nature reviews Clinical oncology 15 (6):366-381. doi:10.1038/s41571-018-0007-1

3. Hanahan D, Coussens LM (2012) Accessories to the crime: functions of cells recruited to the tumor microenvironment. Cancer Cell 21 (3):309-322. doi:10.1016/j.ccr.2012.02.022

4. Rasanen K, Herlyn M (2012) Paracrine signaling between carcinoma cells and mesenchymal stem cells generates cancer stem cell niche via epithelial-mesenchymal transition. Cancer Discov 2 (9):775-777. doi:10.1158/2159-8290.CD-12-0312

5. Lejmi E, Perriraz N, Clement S, Morel P, Baertschiger R, Christofilopoulos P, Meier R, Bosco D, Buhler LH, Gonelle-Gispert C (2015) Inflammatory Chemokines MIP-1delta and MIP-3alpha Are Involved in the Migration of Multipotent Mesenchymal Stromal Cells Induced by Hepatoma Cells. Stem Cells Dev 24 (10):12231235. doi:10.1089/scd.2014.0176

6. Nakamizo A, Marini F, Amano T, Khan A, Studeny M, Gumin J, Chen J, Hentschel S, Vecil G, Dembinski J, Andreeff M, Lang FF (2005) Human bone marrow-derived mesenchymal stem cells in the treatment of gliomas. Cancer Res 65 (8):3307-3318. doi:10.1158/0008-5472.CAN-04-1874 
7. Kim SM, Jeong CH, Woo JS, Ryu CH, Lee JH, Jeun SS (2016) In vivo near-infrared imaging for the tracking of systemically delivered mesenchymal stem cells: tropism for brain tumors and biodistribution. Int $\mathbf{J}$ Nanomedicine 11:13-23. doi:10.2147/IJN.S97073

8. Nwabo Kamdje AH, Kamga PT, Simo RT, Vecchio L, Seke Etet PF, Muller JM, Bassi G, Lukong E, Goel RK, Amvene JM, Krampera M (2017) Mesenchymal stromal cells' role in tumor microenvironment: involvement of signaling pathways. Cancer biology \& medicine 14 (2):129-141. doi:10.20892/j.issn.20953941.2016 .0033

9. Rodini CO, Goncalves da Silva PB, Assoni AF, Carvalho VM, Okamoto OK (2018) Mesenchymal stem cells enhance tumorigenic properties of human glioblastoma through independent cell-cell communication mechanisms. Oncotarget 9 (37):24766-24777. doi:10.18632/oncotarget.25346

10. Ramdasi S, Sarang S, Viswanathan C (2015) Potential of Mesenchymal Stem Cell based application in Cancer. International journal of hematology-oncology and stem cell research 9 (2):95-103

11. Rhee KJ, Lee JI, Eom YW (2015) Mesenchymal Stem Cell-Mediated Effects of Tumor Support or Suppression. Int J Mol Sci 16 (12):30015-30033. doi:10.3390/ijms161226215

12. Mamchur A, Leman E, Salah S, Avivi A, Shams I, Manov I (2018) Adipose-Derived Stem Cells of Blind Mole Rat Spalax Exhibit Reduced Homing Ability: Molecular Mechanisms and Potential Role in Cancer Suppression. Stem Cells 36 (10):1630-1642. doi:10.1002/stem.2884

13. Gilazieva ZE, Tazetdinova LG, Arkhipova SS, Solovyeva VV, Rizvanov AA (2016) Effect of Cisplatin on Ultrastructure and Viability of Adipose-Derived Mesenchymal Stem Cells. BioNanoScience 6 (4):534-539. doi:10.1007/s12668-016-0283-0

14. Galon J, Angell HK, Bedognetti D, Marincola FM (2013) The continuum of cancer immunosurveillance: prognostic, predictive, and mechanistic signatures. Immunity 39 (1):11-26. doi:10.1016/j.immuni.2013.07.008

15. Beckermann KE, Dudzinski SO, Rathmell JC (2017) Dysfunctional T cell metabolism in the tumor microenvironment. Cytokine \& growth factor reviews 35:7-14. doi:10.1016/j.cytogfr.2017.04.003

16. Brand A, Singer K, Koehl GE, Kolitzus M, Schoenhammer G, Thiel A, Matos C, Bruss C, Klobuch S, Peter K, Kastenberger M, Bogdan C, Schleicher U, Mackensen A, Ullrich E, Fichtner-Feigl S, Kesselring R, Mack M, Ritter U, Schmid M, Blank C, Dettmer K, Oefner PJ, Hoffmann P, Walenta S, Geissler EK, Pouyssegur J, Villunger A, Steven A, Seliger B, Schreml S, Haferkamp S, Kohl E, Karrer S, Berneburg M, Herr W, MuellerKlieser W, Renner K, Kreutz M (2016) LDHA-Associated Lactic Acid Production Blunts Tumor Immunosurveillance by T and NK Cells. Cell Metab 24 (5):657-671. doi:10.1016/j.cmet.2016.08.011

17. Roychoudhuri R, Eil RL, Restifo NP (2015) The interplay of effector and regulatory T cells in cancer. Curr Opin Immunol 33:101-111. doi:10.1016/j.coi.2015.02.003

18. Ngambenjawong C, Gustafson HH, Pun SH (2017) Progress in tumor-associated macrophage (TAM)targeted therapeutics. Adv Drug Deliv Rev 114:206-221. doi:10.1016/j.addr.2017.04.010

19. Van Dyken SJ, Locksley RM (2013) Interleukin-4- and interleukin-13-mediated alternatively activated macrophages: roles in homeostasis and disease. Annual review of immunology 31:317-343. doi:10.1146/annurevimmunol-032712-095906

20. Timaner M, Beyar-Katz O, Shaked Y (2016) Analysis of the Stromal Cellular Components of the Solid Tumor Microenvironment Using Flow Cytometry. Current protocols in cell biology 70:19 18 11-19 18 12. doi:10.1002/0471143030.cb1918s70

21. Mingaleeva RN, Solovieva VV, Blatt NL, Rizvanov AA (2013) Application of cell and tissue cultures for potential anti-cancer/oncology drugs screening in vitro. Cellular Transplantation and Tissue Engineering 8 (2):20-28

22. Santo VE, Rebelo SP, Estrada MF, Alves PM, Boghaert E, Brito C (2017) Drug screening in 3D in vitro tumor models: overcoming current pitfalls of efficacy read-outs. Biotechnology journal 12 (1). doi:10.1002/biot.201600505

23. Islamov RR, Rizvanov AA, Mukhamedyarov MA, Salafutdinov, II, Garanina EE, Fedotova VY, Solovyeva VV, Mukhamedshina YO, Safiullov ZZ, Izmailov AA, Guseva DS, Zefirov AL, Kiyasov AP, Palotas A (2015) Symptomatic improvement, increased life-span and sustained cell homing in amyotrophic lateral sclerosis after transplantation of human umbilical cord blood cells genetically modified with adeno-viral vectors expressing a neuro-protective factor and a neural cell adhesion molecule. Curr Gene Ther 15 (3):266-276

24. Kitaeva K, Prudnikov T, Gomzikova M, Tazetdinova L, Soloveva ARV (2017) Analysis of Interactions between CD14+Monocytes, Mesenchymal Stem Cells and HeLa Cells in Double Co-Cultures In Vitro. Blood 130 
25. Rizvanov AA, Yalvac ME, Shafigullina AK, Salafutdinov, II, Blatt NL, Sahin F, Kiyasov AP, Palotas A (2010) Interaction and self-organization of human mesenchymal stem cells and neuro-blastoma SH-SY5Y cells under co-culture conditions: A novel system for modeling cancer cell micro-environment. Eur J Pharm Biopharm 76 (2):253-259. doi:10.1016/j.ejpb.2010.05.012

26. Corkum CP, Ings DP, Burgess C, Karwowska S, Kroll W, Michalak TI (2015) Immune cell subsets and their gene expression profiles from human PBMC isolated by Vacutainer Cell Preparation Tube (CPT) and standard density gradient. BMC immunology 16:48. doi:10.1186/s12865-015-0113-0

27. Pages F, Berger A, Camus M, Sanchez-Cabo F, Costes A, Molidor R, Mlecnik B, Kirilovsky A, Nilsson M, Damotte D, Meatchi T, Bruneval P, Cugnenc PH, Trajanoski Z, Fridman WH, Galon J (2005) Effector memory T cells, early metastasis, and survival in colorectal cancer. The New England journal of medicine 353 (25):2654-2666. doi:10.1056/NEJMoa051424

28. Azimi F, Scolyer RA, Rumcheva P, Moncrieff M, Murali R, McCarthy SW, Saw RP, Thompson JF (2012) Tumor-infiltrating lymphocyte grade is an independent predictor of sentinel lymph node status and survival in patients with cutaneous melanoma. J Clin Oncol 30 (21):2678-2683. doi:10.1200/JCO.2011.37.8539

29. Mahmoud SM, Paish EC, Powe DG, Macmillan RD, Grainge MJ, Lee AH, Ellis IO, Green AR (2011) Tumor-infiltrating CD8+ lymphocytes predict clinical outcome in breast cancer. J Clin Oncol 29 (15):1949-1955. doi:10.1200/JCO.2010.30.5037

30. Gajewski TF, Schreiber H, Fu YX (2013) Innate and adaptive immune cells in the tumor microenvironment. Nat Immunol 14 (10):1014-1022. doi:10.1038/ni.2703

31. Platt JL, Zhou X, Lefferts AR, Cascalho M (2016) Cell Fusion in the War on Cancer: A Perspective on the Inception of Malignancy. Int J Mol Sci 17 (7). doi:10.3390/ijms17071118

32. Bastida-Ruiz D, Van Hoesen K, Cohen M (2016) The Dark Side of Cell Fusion. Int J Mol Sci 17 (5). doi:10.3390/ijms17050638

33. Gast CE, Silk AD, Zarour L, Riegler L, Burkhart JG, Gustafson KT, Parappilly MS, Roh-Johnson M, Goodman JR, Olson B, Schmidt M, Swain JR, Davies PS, Shasthri V, Iizuka S, Flynn P, Watson S, Korkola J, Courtneidge SA, Fischer JM, Jaboin J, Billingsley KG, Lopez CD, Burchard J, Gray J, Coussens LM, Sheppard BC, Wong MH (2018) Cell fusion potentiates tumor heterogeneity and reveals circulating hybrid cells that correlate with stage and survival. Science advances 4 (9):eaat7828. doi:10.1126/sciadv.aat7828

34. Rappa G, Mercapide J, Lorico A (2012) Spontaneous formation of tumorigenic hybrids between breast cancer and multipotent stromal cells is a source of tumor heterogeneity. Am J Pathol 180 (6):2504-2515. doi:10.1016/j.ajpath.2012.02.020

35. Probert C, Dottorini T, Speakman A, Hunt S, Nafee T, Fazeli A, Wood S, Brown JE, James V (2018) Communication of prostate cancer cells with bone cells via extracellular vesicle RNA; a potential mechanism of metastasis. Oncogene. doi:10.1038/s41388-018-0540-5

36. Valadi H, Ekstrom K, Bossios A, Sjostrand M, Lee JJ, Lotvall JO (2007) Exosome-mediated transfer of mRNAs and microRNAs is a novel mechanism of genetic exchange between cells. Nat Cell Biol 9 (6):654-659. doi:10.1038/ncb1596

37. Zhang S, Zhang Y, Qu J, Che X, Fan Y, Hou K, Guo T, Deng G, Song N, Li C, Wan X, Qu X, Liu Y (2017) Exosomes promote cetuximab resistance via the PTEN/Akt pathway in colon cancer cells. Brazilian journal of medical and biological research = Revista brasileira de pesquisas medicas e biologicas 51 (1):e6472. doi:10.1590/1414-431X20176472

38. Chulpanova DS, Kitaeva KV, James V, Rizvanov AA, Solovyeva VV (2018) Therapeutic Prospects of Extracellular Vesicles in Cancer Treatment. Frontiers in immunology 9:1534. doi:10.3389/fimmu.2018.01534

39. Caicedo A, Fritz V, Brondello JM, Ayala M, Dennemont I, Abdellaoui N, de Fraipont F, Moisan A, Prouteau CA, Boukhaddaoui H, Jorgensen C, Vignais ML (2015) MitoCeption as a new tool to assess the effects of mesenchymal stem/stromal cell mitochondria on cancer cell metabolism and function. Sci Rep 5:9073. doi:10.1038/srep09073

40. Smith HA, Kang Y (2013) The metastasis-promoting roles of tumor-associated immune cells. Journal of molecular medicine 91 (4):411-429. doi:10.1007/s00109-013-1021-5

41. Mantovani A, Allavena P, Sica A, Balkwill F (2008) Cancer-related inflammation. Nature 454 (7203):436-444. doi:10.1038/nature07205

42. Poggi A, Zocchi MR (2018) Immunomodulatory properties of mesenchymal stromal cells: still unresolved "Yin and Yang". Current stem cell research \& therapy. doi:10.2174/1574888X14666181205115452

43. Siegel G, Schafer R, Dazzi F (2009) The immunosuppressive properties of mesenchymal stem cells. Transplantation 87 (9 Suppl):S45-49. doi:10.1097/TP.0b013e3181a285b0 